\title{
Comparison of width and depth of maxillary vestibular impression recorded by manual and functional manipulation of borders using modeling plastic and elastomeric material.
}

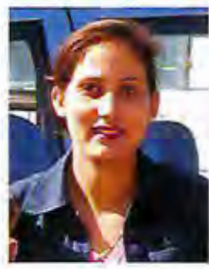

Dr.Harpreet Kaur,

P.G. Student.

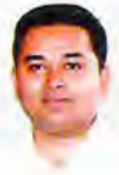

Dr. Shashidhara.H.S,

Professor.

Department of Prosthodontics, CODS, Davangere.

Border molding during impression making is an important step in fabrication of complete dentures. It is a process by which shape of the border of tray is made to conform contours of buccal and labial flanges. The production of an accurate master impression is regarded as an important milestone in fabrication of complete dentures, and depends on skills of clinician, as The purpose of study is to compare the difference in width and depth of vestibular made using modelling plastic and elastomeric impression material.

Methodology

A total of 10 edentulous patients, male or female, with a mean age of 50 years in the age group of 45-60 years were considered for the study.

Procedure

A preliminary impression was made with impression compound for each patient and casts were made with dental plaster. Casts were modified by adding four reference cones made with modeling wax at four differentsites.(Fig1):

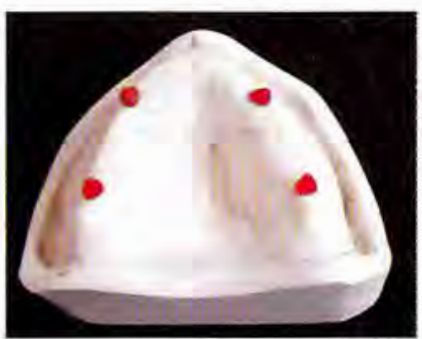

Site 1-Left tuberosity region.

Site 2-Left canine region.

Site 3-Right canine region.

Site 4-Right tuberosiy region.

Cast was duplicated using irreversible hydrocolloid

Impression material. The cones will appear as

Elevation in the duplicated cast.

Fig1: Diagnostic cast with reference cones.

Fabrication of custom trays: Four self cure tray resin custom trays of $2 \mathrm{~mm}$ uniform thickness and $2 \mathrm{~mm}$ short from the reflection of vestibule were fabricated. Cones in the duplicated cast appeared as depressions in custom tray. Two of the custom trays were fabricated with conventional tray handle for border molding by manual manipulation. In the other two trays modified handles that is functional tray handles which were L shaped, $70 \mathrm{~mm}$ in length and $7 \mathrm{~mm}$ in width. (Fig 2)

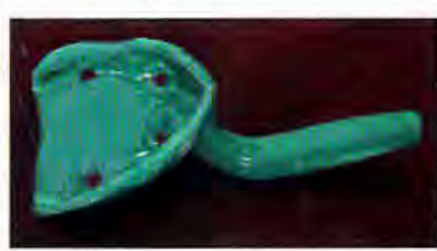

Fig 2: Modified custom tray with functional tray handles

\section{Border molding}

All four trays were checked in patient's mouth, ascertaining that the trays were short by $2 \mathrm{~mm}$ at the periphery and the borders were well rounded with an even thickness of $2 \mathrm{~mm}$.

\section{Border molding by manual manipulation}

The border molding was done section wise. The following border molding movements were carried out;

1.The labial frenum was recorded by raising the lip up and out and pulled down and in and then moving from side to side.

2.The buccal frenum and vestibule was recorded by raising the cheek up and out, and then moved anterioposteriorly.

3.The distobuccal angles of the tray were developed by having the patient swing the mandible fro side to side with the mouth slightly open

4.The posterior palatal seal area recorded by physiologic method along with the Valsalva Maneuver..

The border molding was examined for smooth, and rounded borders of uniform thickness and dull suface. Both green stick compound and elastomer were used to do border molding by manual manipulation in conventional trays, 


\section{Border molding by functional manipulation (Fig 3)}

The border molding was done in a custom tray with modified functional handle.

The following movements were performed;

1. For recording labial frenum and sulcus, the patient was asked to bring the lips down over

the tray handle with their own muscle power and also to pucker their lips and simulate a grin.

2. The buccal borders were recorded by asking the patient to suck and swallow.

3. The patient was then asked to do right and left lateral movements of mandible and close.

4. For recording posterior palatal seal area, the patient was guided and instructe

to tip his head forward to approximately $30^{\circ}$ from vertical position and forcibly place

the tongue against the tray. This maneuver recorded posterior palatal seal in

a functional position.

Once the material was set, the tray was removed from the mouth and the border molding was examined for smooth, and rounded borders of uniform thickness. Excess material flown on tissue surface was removed. Both elastomer and green stick compound were manipulatrd by functional manipulation but molding for elastomer was done in single step.

\section{Preparation of cast}

Completed vestibular impressions were beaded, boxed and poured in dental stone. Reference cones of all four cast had identical contours. Fourty casts were obtained.Profile projector was be used to measure width of the vestibule at the points corresponding to reference cones. Vernier caliper was used to measure the sulcular depth. Time was recorded in minutes.

\section{Results:}

The mean sulcular width measurements were maximum with greenstick compound manipulated by manual manipulation and minimum with condensation silicone manipulated by functional manipulation. The mean sulcular depth was maximum with condensation silicone manipulated by functional manipulation and minimum with green stick by manual manipulation.whereas min time was taken by elastomer by functional manipulation to record the vestibule.

\section{DISCUSSION:}

The present study was carried out to compare the differences in time required, width and depth of vestibular impression made using green stick modeling plastic and condensation silicone impression material in soft putty consistency by functional and manual manipulation of tissues. The specimens were divided into four groups (i.e. Group A to D); study was conducted on ten patients in each group. Group A and B used green stick compound by manual and functional manipulation respectively, Group C and D used condensation silicone in soft putty consistency by manual and functional manipulation of tissues for molding of the vestibular tissues in maxillary edentulous arch.

It was observed that the mean width was maximum with Group A followed by Group C followed by Group B and least width was seen in Group D.

The depth was seen maximum with Group D followed by Group B followed by Group C and least depth was seen in Group A The time taken for border molding by four different groups were recorded and time taken was maximum for Group A followed by Group B followed by Group C and least time was taken by Group D.

C- silicone impression material in soft putty consistency is recently introduced by Zhermack company for border molding The wider and shorter borders seen with modeling compound by manual manipulation could be related to manner in which border molding was performed. When using green stick compound for border molding by manual manipulation, the peripheral tissues were pulled outwards then downwards and inwards. The outwards and downwards manipulation would shorten the flanges and make the borders thicker. The subsequent inward pull will would probably be ineffective in reducing the thickness of borders because of short working time and viscosity of the material

\section{CONCLUSION:}

The basic difference between using modeling plastic and elastomeric impression material for border molding is the difference in working consistencies. Modeling compound being more viscous compared to condensation silicone hence borders obtained were thicker and shorter with modeling compound.Thinner and longer borders seen with elastomer by functional manipulation could be because of the low viscosity of condensation silicone compared to that of modeling compound.

\section{References:}

1) Chaffee NR, Cooper LF, Feito DA. A technique for border molding edentulous impression using vinyl polysiloxane material. Journal of Prosthodontics 1999,8,129-134

2) Tan HK, Hooper PH, Bacrgen CG. Variability in the shape of maxillary vestibular impression recorded with modelling plastic and a polyether impression material. Int Journal of Prosthodontics 1996; 9: 282-289. 